\title{
Modern heuristics for scheduling
}

\author{
Erhan Kozan • Ruhul A. Sarker
}

Published online: 17 May 2011

(C) Springer Science+Business Media, LLC 2011

Scheduling is a common practical decision problem in organizations and businesses. Scheduling is also well-known as a difficult problem area in Operations Research, optimization and computer science domains. Due to the complexity in solving, scheduling problems attracted more attention in the literature than any other optimization problem areas. Although small-scale scheduling problems can be solved optimally using mathematical programming approaches and exact methods, the computational effort required using such methodologies for any medium to large-sized problem is beyond the capability of the current computational resources. This limitation encourages researchers and practitioners to develop case-specific heuristics to solve the problems quickly with reasonable quality of solutions.

Focussing on the latest developments in heuristics for solving scheduling problems, the primary objective of this special issue is to examine the methodological and research issues in solving different scheduling problems. In this issue seven papers have been selected for publication after a thorough peer-review according to the standards of the FSM journal.

The first paper by S. Q. Liu and E. Kozan proposes an approach for solving the coal train scheduling problem that was modeled as a Blocking Parallel-Machine Job-Shop Scheduling problem with additional minor constraints. First, the feasible train schedules were constructed using an innovative constructive algorithm and then a three-stage hybrid algorithm was developed to optimize the train schedules. The method was tested by solving a complex real-world coal rail system in

\footnotetext{
E. Kozan $(\bowtie)$

Mathematical Sciences, Queensland University of Technology, GPO Box 2434, Brisbane, QLD 4001, Australia

e-mail: e.kozan@qut.edu.au
}

\section{R. A. Sarker}

School of Engineering and IT, UNSW@ADFA, Canberra 2600, Australia

e-mail: r.sarker@adfa.edu.au 
Australia. The authors demonstrated that the proposed methodology would be useful for a coal rail system under network and terminal capacity constraints.

The second paper by M. A. B. Amar, H. Camus, T. Bourdeaud'huy and O. Korbaa proposes a mathematical model for solving cyclic job shop scheduling problems with work-in-process minimization. In this paper, the authors introduce several cutting constraints to improve the performances of the model resolution. Several numerical experiments were carried out to assess the relevance of their propositions. The comparison between the proposed model and the model without cut constraints, using a set of benchmarks, shows encouraging improvement for practical implementation.

The next paper by S. M. K. Hasan, R. Sarker, D. Essam, and I. Kacem proposes a DSS for solving job-shop scheduling problems with or without disruptions. In this paper, the authors developed a genetic algorithm with few priority rules and studied job-shop scheduling under process interruptions such as machine unavailability and breakdowns. They showed that the incorporation of priority rules not only improves the solutions but also reduces the overall computational time of the algorithm. The authors also analyzed the effect of machine unavailability and breakdowns on scheduling under different scenarios.

The problem of planning the shunting of train units at a railway workshop area was presented by P. M. Jacobsen and D. Pisinger. The problem involves scheduling the trains to workshops and depot tracks in order to complete the repairs as soon as possible, while avoiding train blockings at the tracks. To solve the problem, the authors presented three heuristic approaches based on Guided Local Search (GLS), Guided Fast Local Search (GFLS) and Simulated Annealing (SA), respectively. They demonstrated the usefulness of the methodologies by solving realistic problem instances. It was shown that both GLS and SA find solutions within few minutes which are close to the best MIP solutions.

A scheduling approach for optimizing sugarcane rail operations is proposed by M. Masoud, E. Kozan and G. Kent. The sugarcane transport system is very complex using a daily schedule and consisting of a set of locomotives runs to satisfy the requirements of the mill and harvesters. In this paper, the sugarcane rail operations are defined as a blocking job shop scheduling problem. Both mixed-integer programming and constraint programming search techniques are integrated for solving the problem. A case study is solved to test the approach and to show the applicability of the proposed approach.

The sixth paper by V. A. Shim, K. C. Tan, J. Y. Chia, and J. K. Chong studied the application of two evolutionary algorithms for solving a bi-objective travelling salesman problem. The problem is to find the order of the cities by simultaneously minimizing the travelling distance and the travelling cost. These two algorithms (EDA and GA) are then combined to form a hybrid algorithm in order to complement the limitations of both algorithms. The authors showed that the hybrid algorithm is capable of finding a set of good trade-off solutions.

The final paper by M. Kharbeche, J. Carlier, M. Haouari, and A. Moukrim investigates an exact method for the Robotic Cell Problem. The authors present a branch-and-bound algorithm as well as a genetic algorithm for solving the problem. They provide evidence that medium-sized instances with up to 176 operations can 
be solved optimally and make the case that the genetic algorithm delivers good solutions while requiring short CPU times. They also derived new lower bounds that outperform all other lower bounds appeared in the literature.

The special issue has greatly benefited from the cooperation among the authors, reviewers, and editors. We would like to express our sincere thanks to the reviewers for their excellent and timely refereeing, and to the editor-in-chief for timely help and guidance. Last, but not least, we thank all authors for their contributions which made this special issue possible. 